\title{
Flow Visualization in Out-of-Round Rocket Nozzles
}

\author{
C. Génin ${ }^{1}$, S. Jack ${ }^{2}$, R. Stark ${ }^{1}$ \\ ${ }^{1}$ Institute of Space Propulsion, German Aerospace Center (DLR),Lampoldshausen, Germany \\ ${ }^{2}$ Institute of Aerodynamics and Flow Technologiy, German Aerospace Center \\ (DLR),Braunschweig, Germany
}

Corresponding Author: chloe.genin@dlr.de

\section{INTRODUCTION}

Flow fluctuations in or around rocket nozzles can lead to a deformation of their structure. In case of separated nozzle flow, an inward bended nozzle wall shifts the flow separation position downstream, increasing the bending force lever arm. This flow structure interaction is a selfreinforcing effect, which can potentially lead to heavy structural damages. These disturbances can be produced either by combustion instabilities or ambient pressure fluctuations. Under certain conditions, they may excite the bell nozzle eigenfrequency. As a result, the structure responds and the nozzle ovalizes. For this reason, the investigation of flow-structure interaction in ovalized nozzles under separated flow condition is difficult to realize ${ }^{(1,2,3,4)}$.

In order to understand and study these phenomena, a collaborative work between the institutes of Space Propulsion and Aerodynamics and Flow Technology of the German Aerospace Center (DLR) has been started in the framework of in-house project ProTAU. A step by step, both numerical and experimental investigation is carried out on nozzle ovalization. The experimental work is also part of the special research field SFB Transregio 40 collaborative work. The study started with the validation of the numerical method to simulate separated flow in axisymmetric conventional nozzles. Based on three initial non-deformed nozzles, seven different out-of-round geometries have been defined. All of these permanently deformed nozzles were numerically simulated and three geometries were selected out of them for experimental investigation, after the simulation had shown the measurability of the flow behavior within the facility capability. Once the numerical method will be fully validated, it will be implemented to investigate flow-structure interaction in out-of-round nozzles.

\section{TRUNCATED IDEAL NOZZLE EXHAUST FLOW}

The present study focuses on the deformation of truncated ideal contour (TIC) nozzles. The homogenous flow without internal shock of a TIC nozzle facilitates the research of contour deformation effects on the flow. For the purpose of this study, three initial non deformed contours have been chosen. They present design Mach number of 4.8, 5.3 and 5.8 and are designated resp. as TIC-2048, TIC-2053 and TIC-2058. The contours share the same subsonic geometry and a throat radius of $10 \mathrm{~mm}$. Each one of them was truncated to a length allowing full flowing
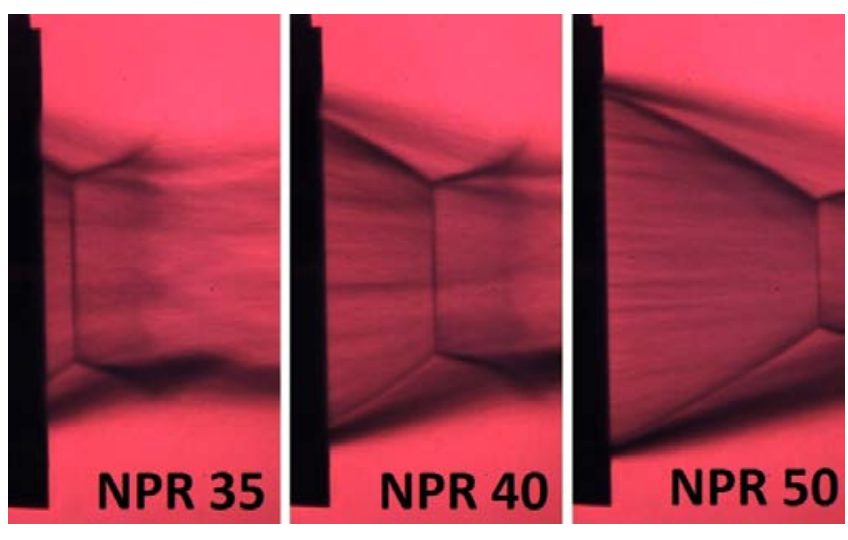

Figure 1: Shock system out of non-deformed TIC-2048 nozzle for various NPR values 
condition at a nozzle pressure ratio (NPR, total over ambient pressure) of 50, corresponding to the maximal value reached at test bench P6.2. The models were also tested with the same methodology in order to verify the measurement methods and to yield validation data for the accompanying CFD study. Figure 1 illustrates an example of the schlieren pictures taken out of the initial non-deformed TIC-2048. The measurements are not part of the present work, but can be found in previous publications by the authors ${ }^{(5)}$.

\section{OVALIZATION PRINCIPLE}

The initial contours were deformed into seven out-of-round nozzle contours using various methods. The deformations were chosen to provide a parameter study, with variation of the deformation function and amplitude, the deformation start position and the initial contour.

The cross section of the deformed contours features an ellipse. The different deformations are summarized in Table 1. Geometries 1 to 3 figure the deformations of contour TIC-2048 with variations of the deformation amplitude, resp. $5 \%$ to $15 \%$. The amplitude is given as a comparison between the initial nozzle end radius and the obtained major axis after deformation. Geometries 2, 4 and 7 feature the same deformation applied to the three initial contours, TIC2048, TIC-2053 and TIC-2058. Geometries 4 to 6 are variations of contour TIC-2053, whereas 4 and 5 are deformed with a different starting point and 4 and 6 following different functions.

Table 1: Deformation example of a table.

\begin{tabular}{|l|c|c|c|l|}
\cline { 2 - 5 } \multicolumn{1}{c|}{} & $\begin{array}{c}\text { Initial } \\
\text { contour }\end{array}$ & Amplitude & $\begin{array}{c}\text { Start } \\
\text { point }\end{array}$ & Function \\
\hline Geometry 1 & TIC-2048 & $5 \%$ & $\varepsilon=5$ & polynomial \\
\hline Geometry 2 & TIC-2048 & $10 \%$ & $\varepsilon=5$ & polynomial \\
\hline Geometry 3 & TIC-2048 & $15 \%$ & $\varepsilon=5$ & polynomial \\
\hline Geometry 4 & TIC-2053 & $10 \%$ & $\varepsilon=5$ & polynomial \\
\hline Geometry 5 & TIC-2053 & $10 \%$ & $\varepsilon=1$ & polynomial \\
\hline Geometry 6 & TIC-2053 & $10 \%$ & $\varepsilon=5$ & linear \\
\hline Geometry 7 & TIC-2058 & $10 \%$ & $\varepsilon=5$ & polynomial \\
\hline
\end{tabular}

A preliminary CFD study has been conducted in the framework of the DLR project ProTAU to verify the feasibility of this experimental investigation and to optimize the measurement methodology. Out of this study, three geometries have been chosen for further investigation and have been manufactured for test. The models realized out of geometries 2, 3 and 4 and tested in this study are represented in Fig. 1, left.

\section{EXPERIMENTAL SETUP}

The test campaign was conducted at P6.2 cold flow facility at DLR Lampoldshausen. The dry nitrogen feeding line provides mass flow up to $4 \mathrm{~kg} / \mathrm{s}$ with a total pressure up to $5 \mathrm{MPa}$. The nozzles were mounted on the horizontal rig (see figure 2, right) and tests were conducted under ambient conditions. The feeding pressure was progressively varied up and down with a constant gradient of $+/-0.2 \mathrm{MPa} / \mathrm{s}$, leading to the displacement of the separation point along the nozzle contour until full flowing condition. Wall pressure ports placed in line within small axial intervals of $4 \mathrm{~mm}$ permit to follow the position of the separation point. The ports were also regularly distributed in circumferential direction. Five rows were positioned between major and 
minor axis with an angle of $22.5^{\circ}$, each one featuring nineteen positions, for a total of 95 wall pressure ports for each nozzle.
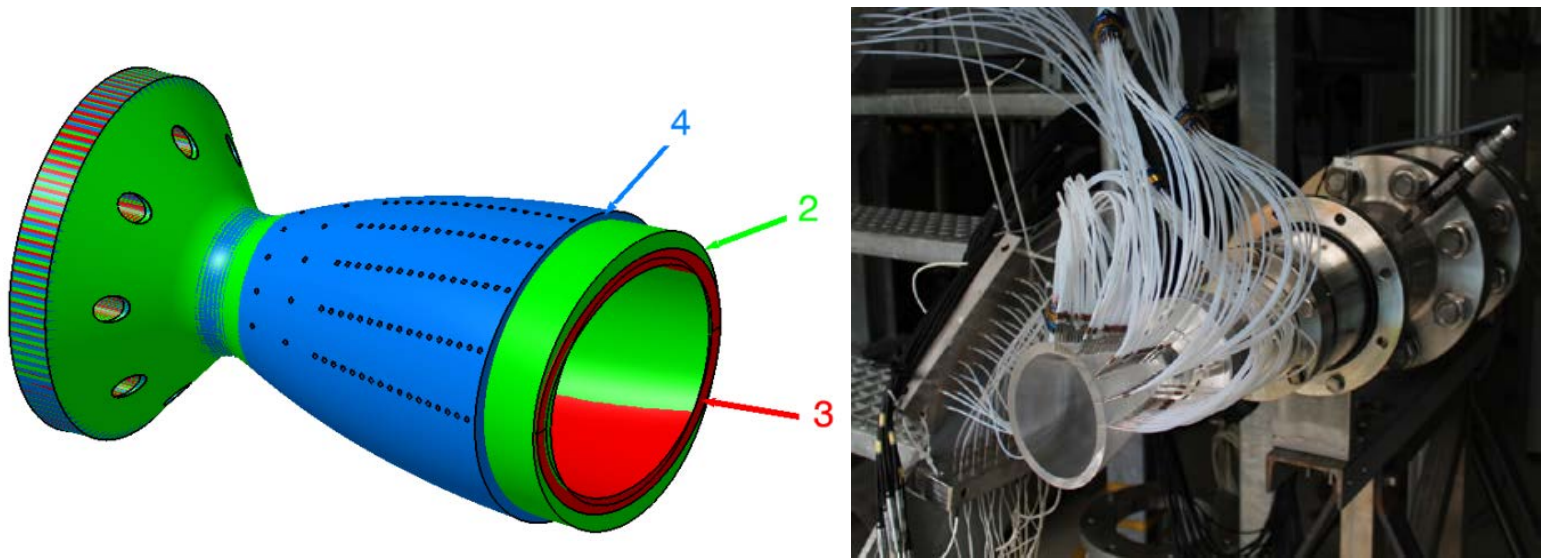

Figure 2: Sketch of the three nozzle geometries (left) and out-of-round nozzle model 4 mounted on the horizontal test position at P6.2 facility, in minor axis view (right).

The evolution of the shock system outside of the nozzle models was recorded with schlieren optics in Z-configuration. As the shock system was expected not to be axisymmetric the models were rotated around their own axis. The exhaust flow of each model was recorded in four positions, corresponding to four circumferential angles: in the direction of the major axis $\left(0^{\circ}\right)$, a rotation of $+45^{\circ}$ and $-45^{\circ}$ and in the direction of the minor axis $\left(-90^{\circ}\right)$.

\section{RESULTS}

Figure 3 gives an example of the shock system out of nozzle model 4 observed with an angle of $+45^{\circ}$ for the four NPR values 35, 40, 45 and 50. With increasing value of the NPR, the separation point moves downstream the nozzle contour and the shock system is shifted out of the nozzle. The Mach disk appears in the $45^{\circ}$ view in " 8 " shape, particularly for low NPR values. The classical Mach disk known from axisymmetric nozzle exhaust flow is in a deformed nozzle bended into a saddle shape.
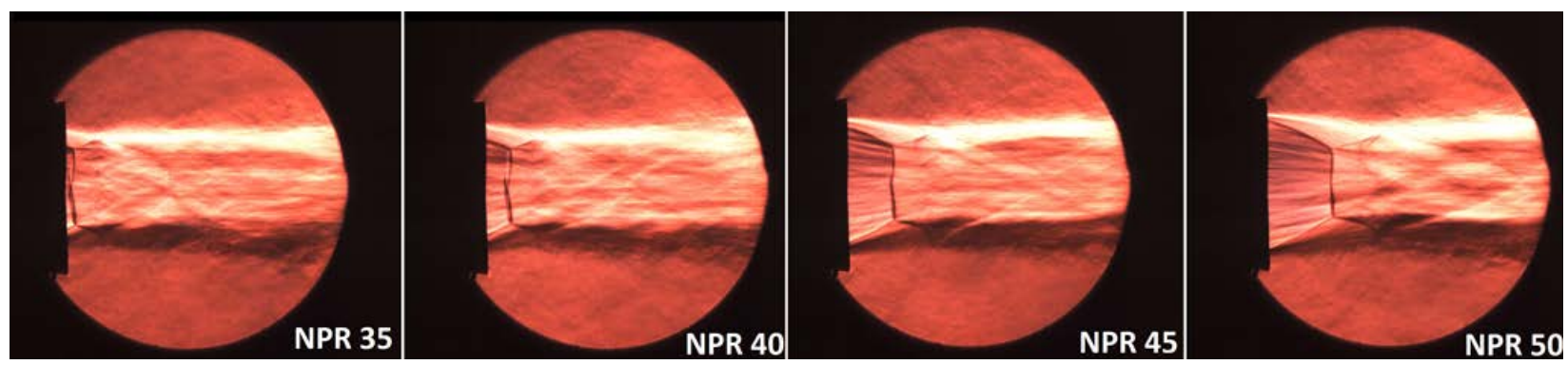

Figure 3: Shock system out of geometry 4 for various NPR values.

The schlieren pictures were recorded for each nozzle in the four observation angles, while the exact same feeding pressure up- and down-ramping was conducted. In this manner, for each NPR value, the shock system is available in various angles. The pictures in Fig. 4 represent the exhaust flow out of geometry 3 (15\% deformation) in four views. The shock system was recorded for NPR 40, corresponding to a flow separation position from the wall situated near the nozzle end. 
The pictures are labelled as follow: picture a) represents the shock system viewed in the major axis view, d) in the minor axis view and pictures b) and c) are in the $+/-45^{\circ}$ directions views. Geometry 3 has been chosen to illustrate the effect of contour ovalization on flow behavior as it presents a deformation amplitude of $15 \%$, which is the highest deformation value for the tested nozzle models. The general behavior to deformation is comparable but the shock system variations are more visible with higher ovalization amplitude.
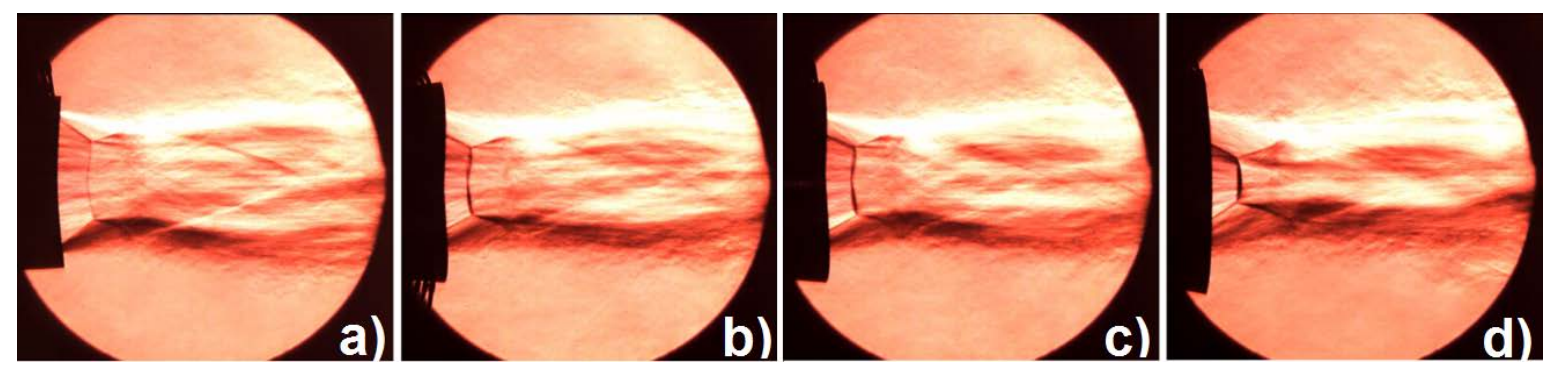

Figure 4: Schlieren pictures of the exhaust flow out of geometry 3 for NPR 40, a) in major axis view, b) and c) in $+/-45^{\circ}$ view and d) in minor axis view.

Positions along the shock system were collected for each picture. In Fig. 5, the shock system out of geometry 3 has been reconstituted out of these measurements for three NPR values, 35, 40 and 50. The position of the separation line was interpolated as the intersection of separation shock and wall contour. The deformation of the Mach disk into a Mach saddle is visible in this 3dimensinal representation, particularly for NPR 35 (blue shock system) and NPR 50 (red shock system).

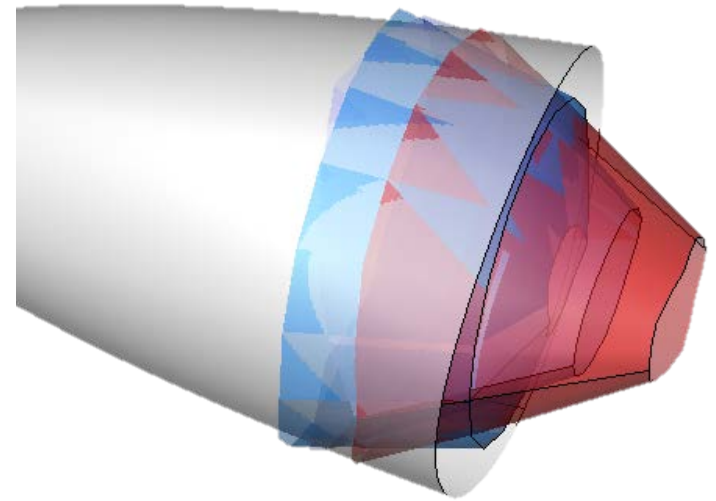

Figure 5: Reconstitution of the shock system out of geometry for NPR 35, 40 and 50.

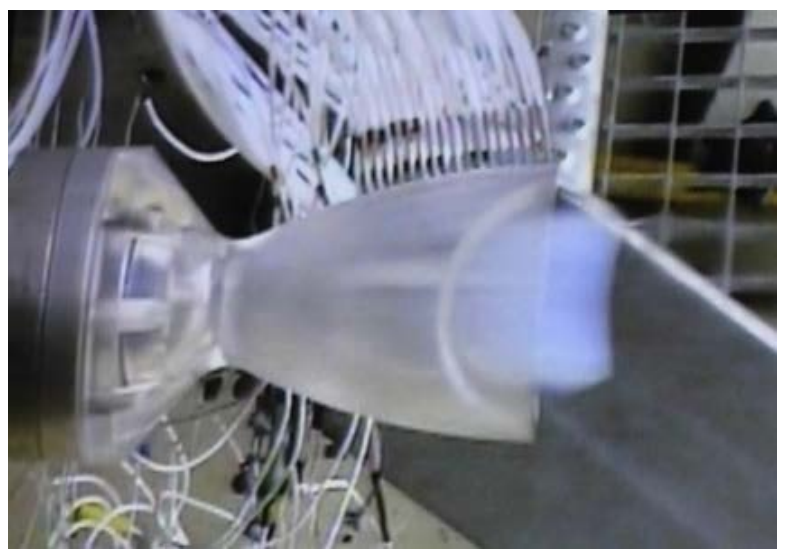

Figure 6: Picture of geometry 4 model during test at P6.2 bench.

Figure 6 is a picture taken during down-ramping of a test on geometry 4 model (NPR 42). The cold nitrogen has condensate due to the low temperature and become visible upstream the shock system. It can be seen that the Mach saddle is bended inward. In addition the separation line is visible on the nozzle wall. The models were made out of PMMA (acrylic glass) and are transparent. During down-ramping, the separation line moves up-stream, leaving the nozzle wall very cold. The back flow is sucked into the nozzle and the air humidity froze directly downstream the separation line, as visible in figure 6. The nozzle is observed in the major axis line of sight. 
The separation point is still at nozzle end on the minor axis (vertical direction), whereas it lies far up-stream on the major axis (horizontal direction).

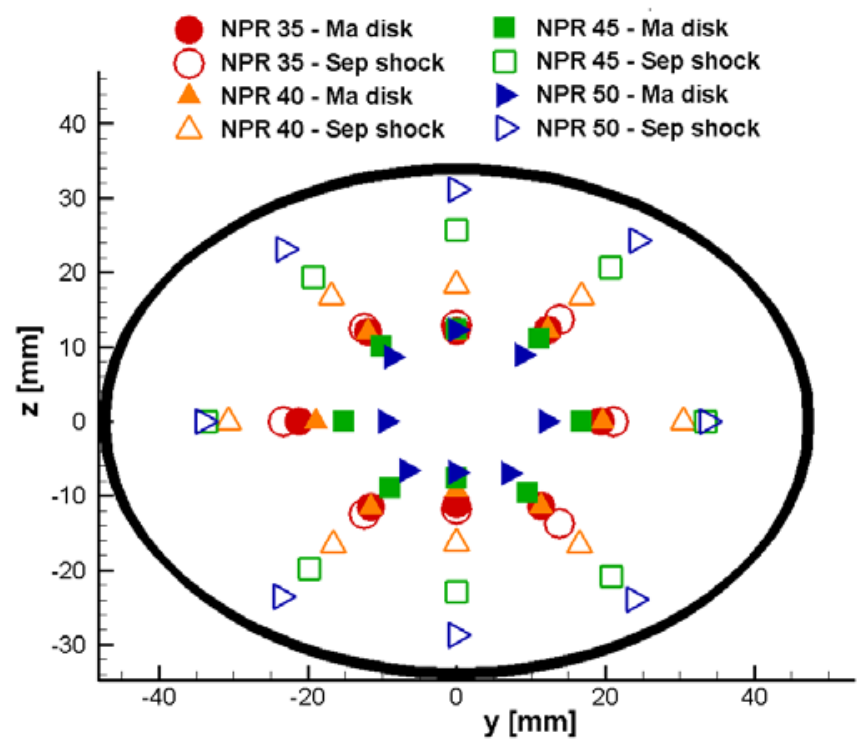

Figure 7: Position of the triple point and the intersection of separation shock and nozzle exit cross section for various NPR values in geometry 3.

Figure 7 illustrates the evolution of the triple points (filled symbols) and of the intersection point between oblique separation shock and nozzle exit cross section (plain symbols) in geometry 3. At low NPR both symbols are close to one another. The Mach "saddle" is in the vicinity of the nozzle exit cross section. The symbols describe an ellipse, comparable to the shape of the ovalized nozzle exit. With increasing NPR values the separation point moves downstream the wall toward the nozzle end. The intersection of separation shock and nozzle exit is consequently closer to the nozzle wall, as can be seen for NPR 45 and 50 in figure 7. The shape of the intersection line evolves from a small ellipse into a large circle. The shape of the Mach saddle follows a similar trend: the elliptic Mach disk at NPR 35 changes into a circular shape at NPR 50. As the shock system moves further downstream the dimensions of the Mach saddle decreases. As the NPR increases the shock system out of an ovalized nozzle contour behave more and more like the shock system out of a conventional axisymmetric nozzle contour.

\section{CONCLUSIONS}

Three stiff ovalized nozzle models have been tested under cold flow conditions. The shock system out of the models was recorded with schlieren optics. The pictures show the evolution of the classical Mach disk into a Mach "saddle”. The deformation decreases with increasing nozzle pressure ratio. The observation of the shock system from different angles has permitted to get a sense of its three dimensional structure. The experimental results obtained in this study will be used as validation data for the numerical investigation of nozzle ovalization. The next step would be to investigate the flow reaction to an applied deformation of the contour.

\section{AKNOWLEDGEMENTS}

This work was co-funded by the special research field SFB-TRR40 of the Deutsche Forschungsgemeinschaft (DFG). 


\section{REFERENCES}

1. Tuovila J., Land S. Experimental study of aeroelastic instability of overexpanded rocket nozzle extensions. NASA-TN-D-4471, Langley Research Center, (1968).

2. Brown M., Ruf J., Reed D., D’Agostino M., Keanini R. Characterization of Side Load Phenomena Using Measurement of Fluid / Structure Interaction. AIAA 2002-3999, 38th AIAA Joint Propulsion Conference, Indianapolis, IN, (2002).

3. Moreaux N., Girard S. Experimental Assessment of Aeroelastic Coupling in a Rocket Engine Nozzle. ONERA TP 2003-80, IFASD, Amsterdam, Netherlands, (2003).

4. Xia Y., Schwane R. Investigation of the Aerodynamic Influence of a Non-Axisymmetric Flow on Side Load Generation in Over-Expanded Rocket Nozzles, DGLR-JT2004-274, (2004).

5. Génin C., Stark R., Jack S. Flow Separation in Out-of-Round Nozzles, a Numerical and Experimental Study, Progress in Flight Physics, Vol. 7, Springer, (2015). 